\title{
Periodic Solution for Vibration of Euler-Bernoulli Beams Subjected to Axial Load Using DTM and HA
}

\author{
Mohammadreza Azimi ${ }^{*}$ and Saeed Kariman²
}

${ }^{1}$ Faculty of Engineering, Tarbiat Modares University, Iran

${ }^{2}$ Assistant Professor, Faculty of Engineering, Tarbiat Modares University, Tehran, Iran

\begin{abstract}
This paper is concerned with analytical approximate solutions, to the nonlinear vibration of Euler-Bernoulli beams subjected to the axial loads. Hamltonian Approach (HA) and Differential Transformation Method (DTM) which are applied to solve the nonlinear differential equation cause in current problem and consequently the relationship between the natural frequency and the initial amplitude is obtained in an analytical form. To verify the accuracy of the present approach, illustrative examples are provided and compared with Exact Solution. The procedure yields rapid convergence with respect to the exact integral solution.
\end{abstract}

Keywords: Nonlinear oscillation; Euler-Bernoulli beam; Hamiltonian approach; Differential transformation method

\section{Introduction}

Beams have wide applications in Engineering Structures and many Structures such as long span bridges, Aircraft wings, flexible satellite can be modeled to as flexible beams [1]. Large amplitude vibration of beams usually leads to nonlinearity. The nonlinear vibrations of free classical Euler Bernoulli beams have been investigated in pervious researches both numerically and analytically $[2,3]$.

Nonlinear problems such as vibration of beams can be solved numerically and analytically, but obtaining analytical solution for nonlinear systems is very important due to limitations of numerical methods [4]. With the rapid development of nonlinear science, there appears an ever-increasing interest of scientists and engineers in the analytical asymptotic techniques for nonlinear problems. In the recent years, many asymptotic techniques including the Energy Balance Method (EBM) [5], Adomian Decomposition Method (ADM) [6], Hamiltonian Approach (HA) [7], Differential transformation method (DTM) [8,9], Parametrized Perturbation Method (PPM) [10], Amplitude Frequency Formulation (AFF) [11] and Variational Iteration Method (VIM) [12] have been developed to construct many types of aproximate solutions of nonlinear differential equations.

The aim of this paper was to determine the periodic solutions to nonlinear Euler Bernoulli beam subjected to axial load by applying HA and DTM. By comparing the analytical results with exact solutions, we illustrated the high accuracy of these methods.

\section{Mathematical formulation}

Euler-Bernoulli beam theory assumes that plane cross sections, normal to the natural axis before deformation, continue to remain plane and continue to remain normal to the neutral axis and do not undergo any strain in their planes [3].

The nonlinear partial differential equation of the beam when the effects of mid-plane stretching are not negligible can be written as follows:

$$
E I \frac{\partial^{4} \hat{w}}{\partial \hat{x}^{4}}+m \frac{\partial^{2} \hat{w}}{\partial \hat{t}^{2}}-N_{0} \frac{\partial^{2} \hat{w}}{\partial \hat{x}^{2}}+\frac{E A}{2 L} \int_{0}^{L}\left(\frac{\partial \hat{w}}{\partial \hat{x}}\right)^{2} d x \frac{\partial^{2} \hat{w}}{\partial \hat{x}^{2}}=0,
$$

Where $E$ is the Young's modulus of elasticity of the beam material, $I$ is the second moment of area of the cross section with respect to the bending axis, $\hat{w}$ is the beam deflection, $m$ is the longitudinal density, $L$ is the length of the beam, $\hat{t}$ is the time, $A$ is the cross sectional area of the beam and $N_{0}$ is the pretension of the beam.

For convenience, the following non-dimensional variables are used:

$$
x=\frac{\hat{x}}{L}, w=\frac{\hat{w}}{r}, t=\sqrt{\frac{m L^{4}}{\beta^{4} E I}}, r=\sqrt{\frac{I}{A}},
$$

Assuming $W(x, t)=\varphi(x) \psi(t)$ where $\varphi(x)$ is the first eigenmode of the beam [9] and applying the Galerkin method, the equation of motion is obtained as following form:

$$
\begin{aligned}
& \ddot{\phi}+\omega_{0}^{2} \phi+\alpha \phi^{3}=0 \\
& \phi(0)=\frac{W_{\max }}{L}, \bar{\phi}(0)=0
\end{aligned},
$$

In equation. $3 \omega_{0}^{2}$ and $\alpha$ are defined as follows:

$$
\begin{aligned}
& \omega_{0}^{2}=\frac{1}{\beta^{4} F_{1}}\left(F_{3}+\frac{N_{0}}{E I}\left(\frac{L}{r}\right)^{2} F_{2}\right), \\
& \alpha=\frac{1}{2 \beta^{4} F_{1}}\left(\frac{L}{r}\right)^{2} F_{2}^{2}
\end{aligned}
$$

Where

$$
F_{1}=\int_{0}^{1} \phi^{2} d x, F_{2}=\int_{0}^{1} \phi^{\prime 2} d x, \quad F_{3}=\int_{0}^{1} \phi^{\prime \prime} d x,
$$

The nonlinear ordinary differential equation in equation. 3 is the governing nonlinear vibration of Euler-Bernoulli beams.

*Corresponding author: Mohammadreza Azimi, Faculty of Engineering, Tarbiat Modares University, Iran, E-mail: M_R_Azimi1991@yahoo.com

Received June 11, 2013; Accepted August 28, 2013; Published August 30, 2013

Citation: Azimi M, Kariman S (2013) Periodic Solution for Vibration of EulerBernoulli Beams Subjected to Axial Load Using DTM and HA. J Appl Mech Eng 2: 125. doi:10.4172/2168-9873.1000125

Copyright: @ 2013 Azimi M, et al. This is an open-access article distributed under the terms of the Creative Commons Attribution License, which permits unrestricted use, distribution, and reproduction in any medium, provided the original author and source are credited. 


\section{Solution procedure}

In this section the solution procedure of nonlinear vibration of Euler Bernoulli beam subjected to axial load by using DTM and HA will be presented.

Basic idea of DTM: Let $x(t)$ be analytic in a domain $D$ and let $t=t_{i}$ represents any point in $D$. The function $x(t)$ is then represented by one power series whose center is located at $t_{i}$. The Taylor series expansion function of $x(t)$ is in the form of:

$$
x(t)=\sum_{k=0}^{\infty} \frac{\left(t-t_{i}\right)}{k !}\left[\frac{d^{k} x(t)}{d t^{k}}\right]_{t=t_{i}} \quad \forall t \in D,
$$

The differential transformation of the function $x(t)$ can be defined as following form [9]:

$$
X(k)=\sum_{k=0}^{\infty} \frac{H^{k}}{k !}\left[\frac{d^{k} x(t)}{d t^{k}}\right]_{t=0},
$$

Where $x(t)$ is the original function and $X(k)$ is the transformed function. The differential inverse transform of $X(k)$ is defined as follows:

$$
x(t)=\sum_{k=0}^{\infty}\left(\frac{t}{H !}\right)^{k} X(k),
$$

It is clear that the concept of the differential transformation is based upon the Taylor series expansion. The values of function $X(k)$ at values of argument $k$ are referred to as discrete, i.e. $X(0)$ is known as the zero discrete, $X(1)$ as the first discrete, etc. the function is expressed by a finite series and equation. 8 can be written as:

$$
x(t)=\sum_{k=0}^{n}\left(\frac{t}{H !}\right)^{k} X(k),
$$

Mathematical operations performed by DTM are listed in Table 1.

Basic idea of HA: Consider a simple second order conservative oscillator with odd-nonlinearity in the form:

$$
\ddot{u}=f(u), \quad u(0)=A, \quad \dot{u}(0)=0,
$$

where a dot denotes differentiation with respect to $t$ and $f(-u)=-f(u)$. The Hamiltonian of the nonlinear oscillation equation can be written as following form:

$$
H=\frac{\dot{u}^{2}}{2}+F(u)=\text { cons. , }
$$

In Equation.11 $\frac{\dot{u}^{2}}{2}$ and $F(u)$ denote kinetic and potential energy, respectively. Therefore, we can write:

\begin{tabular}{|c|c|}
\hline Original Function & Transformed Function \\
\hline$x(t)=\alpha f(t) \pm \beta g(t)$ & $X(k)=\alpha F(k) \pm \beta G(k)$ \\
$x(t)=f(t) g(t)$ & $X(k)=\sum_{l=0}^{k} F(l) G(k-l)$ \\
$x(t)=\frac{d f(t)}{d t}$ & $X(k)=(k+1) F(k+1)$ \\
$x(t)=\frac{d^{2} f(t)}{d t^{2}}$ & $X(k)=(k+1)(k+2) F(k+2)$ \\
\hline
\end{tabular}

Table 1: Some of the basic operations of Differential Transformation Method. $\frac{\partial H}{\partial A}=0$

We can introduce $\tilde{H}(u)$ as follows:

$\tilde{H}(u)=\int_{0}^{\frac{T}{4}}\left(\frac{\dot{u}^{2}}{2}+F(u)\right) d t=\frac{T H}{4}$,

So,

$\frac{\partial}{\partial A}\left(\frac{\partial \tilde{H}}{\partial T}\right)=0$,

Or,

$\frac{\partial}{\partial A}\left(\frac{\partial \tilde{H}}{\partial(1 / \omega)}\right)=0$,

Consequently, the approximate frequency can be found from Equation 15.

Application of DTM: Taking the differential transform of equation 3

$$
\begin{aligned}
& (k+1)(k+2) \Phi(k+2)+\omega_{0}^{2} \Phi(k) \\
& +\alpha \sum_{m=0}^{k} \Phi(k-m) \sum_{l=0}^{m} \Phi(l) \Phi(m-l)=0
\end{aligned},
$$

From boundary conditions in equation 3 , that we have it in point $t=0$ and exerting transformation:

$$
\begin{aligned}
& \Phi(0)=A \\
& \Phi(1)=0
\end{aligned}
$$

We will have:

$$
\begin{aligned}
& \Phi(2)=-\frac{\omega_{0}^{2}}{2}-\frac{\alpha}{2} \\
& \Phi(3)=0 \\
& \Phi(4)=-\frac{\omega_{0}^{4}}{24}+\frac{\alpha \omega_{0}^{2}}{6}+\frac{\alpha^{2}}{8} \\
& \Phi(5)=0 \\
& \Phi(6)=-\frac{\omega_{0}^{6}}{720}-\frac{5 \alpha \omega_{0}^{4}}{144}-\frac{17 \alpha^{2} \omega_{0}^{2}}{240}-\frac{3 \alpha^{3}}{80} \\
& \Phi(7)=0 \\
& \Phi(8)=\frac{\omega_{0}^{8}}{40320}+\frac{13 \alpha \omega_{0}^{6}}{2520}+\frac{47 \alpha^{2} \omega_{0}^{4}}{2240}+\frac{3 \alpha^{3} \omega_{0}^{2}}{112}+\frac{7 \alpha^{4}}{640}
\end{aligned}
$$

The above process is continuous. Substituting equation 18 into the main equation based on DTM, it can be obtained that the closed form of the solutions is:

$$
\Phi(t)=\Phi(0) \times t^{0}+\Phi(1) \times t^{1}+\Phi(2) \times t^{2}+\cdots,
$$

The approximate analytical solution can be easily yield.

Application of HA: Considering the following equation:

$$
\begin{aligned}
& \ddot{\phi}+\omega_{0}^{2} \varphi+\alpha \phi^{3}=0 \\
& \phi(0)=A, \quad \dot{\phi}(0)=0
\end{aligned}
$$

Its Hamiltonian can be easily obtained: 
$H=-\frac{\dot{u}^{2}}{2}+\frac{\omega_{0}^{2}}{2} u^{2}+\frac{\alpha}{4} u^{4}$

Inserting equation.21 with respect to $t$ from 0 to $T / 4$, we yield:

$\tilde{H}=\int_{0}^{\frac{T}{4}}\left(-\frac{\dot{u}^{2}}{2}+\frac{\omega_{0}^{2}}{2} u^{2}+\frac{\alpha}{4} u^{4}\right) d t$

Considering the initial conditions, we assume that the solution can be expressed as:

$$
\phi(t)=A \cos (\omega t)
$$

Substituting it into equation.3, we have:

$$
\tilde{H}=\int_{0}^{\frac{T}{4}}\left(\frac{1}{2} A^{2} \sin (\omega t)^{2} \omega^{2}+\frac{\omega_{0}^{2}}{2} A^{2} \cos (\omega t)^{2}+\frac{\alpha}{4} A^{4} \cos (\omega t)^{4}\right)
$$

Therefore,

$$
\begin{aligned}
& \tilde{H}=\int_{0}^{\frac{\pi}{2}}\left(\frac{1}{2} A^{2} \sin (\phi)^{2} \omega+\frac{\omega_{0}^{2}}{2 \omega} A^{2} \cos (\phi)^{2}+\frac{\alpha}{4 \omega} A^{4} \cos (\phi)^{4}\right) d \phi \\
& =\frac{1}{64} \frac{\pi\left(8 \omega_{0}^{2} A^{2}+3 A^{2} \alpha+8 A^{2} \omega^{2}\right)}{\omega}
\end{aligned}
$$

According to the Hamiltonian approach, we set:

$$
\frac{\partial}{\partial A}\left(\frac{\partial \tilde{H}}{\partial(1 / \omega)}\right)=-\frac{1}{4} A \pi \omega_{0}^{4}+\frac{1}{4} A \pi \omega_{0}^{2}+\frac{3}{16} A^{3} \pi \alpha
$$

So, we can easily obtain the following approximate frequencyamplitude relationship:

$$
\omega_{H A}=\frac{1}{2} \sqrt{3 \alpha A^{2}+4 \omega_{0}^{2}}
$$

The approximate analytical solution using Hamiltonian Approach is:

$$
\phi_{H A}=A \cos \left(\left(\frac{1}{2} \sqrt{3 \alpha A^{2}+4 \omega_{0}^{2}}\right) t\right)
$$

\section{Results and Discussions}

We compared the results of the present analysis based on DTM with an exact integral solution and absolute error, as demonstrated in Table 2 for case of $\alpha=0.5, \omega_{0}^{2}=0.5$ and $A=1$.

A very interesting agreement between the results is observed too, which confirms the excellent validity of the Differential Transformation Method (DTM).

\begin{tabular}{|c|c|c|c|}
\hline $\mathbf{t}$ & Exact & DTM & Absolute \\
\cline { 3 - 4 } & & & Error \\
\hline 0 & 1 & 1 & 0 \\
\hline 0.25 & 0.969071 & 0.972781 & 0.003709 \\
\hline 0.5 & 0.879941 & 0.892604 & 0.012663 \\
\hline 0.75 & 0.74226 & 0.763835 & 0.02157 \\
\hline 1 & 0.568569 & 0.593484 & 0.024915 \\
\hline 1.25 & 0.371179 & 0.390821 & 0.019646 \\
\hline 1.5 & 0.160398 & 0.166891 & 0.006491 \\
\hline 1.75 & -0.055622 & -0.066131 & 0.010507 \\
\hline 2 & -0.269864 & -0.295551 & 0.025686 \\
\hline
\end{tabular}

Table 2: Comparison of DTM results and Exact Solution when, $\alpha=0.5, \omega_{0}^{2}=0.5, A=1$.

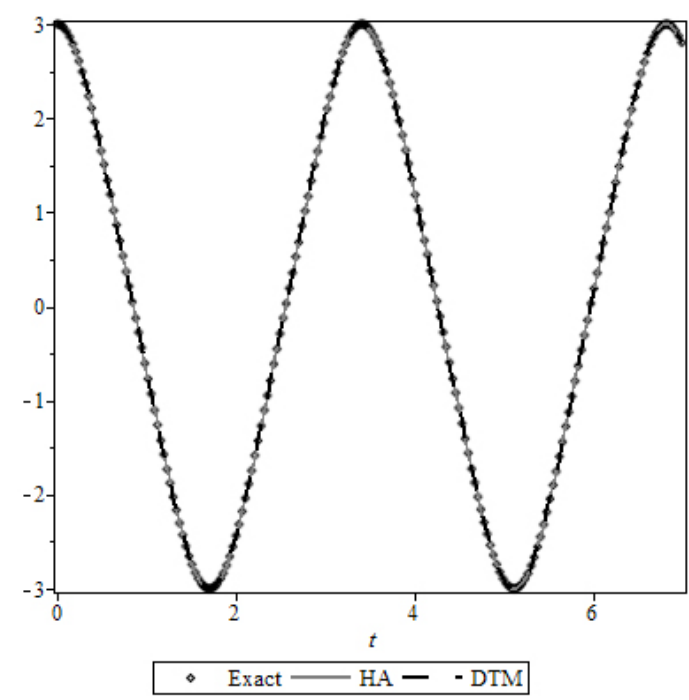

Figure 1: Analytical solutions and exact ones for case of $\alpha=0.15, \omega_{0}^{2}=0.5, A=3$.

\begin{tabular}{|c|c|c|c|}
\hline$\alpha$ & $\omega_{0}^{2}$ & $T_{\text {exact }}$ & $T_{H A}$ \\
\hline \multirow{4}{*}{0.25} & 0.5 & 7.9534 & 7.9476 \\
\cline { 2 - 4 } & 1 & 6.3006 & 6.2831 \\
\cline { 2 - 4 } & 2 & 4.7381 & 4.7497 \\
\hline \multirow{3}{*}{0.5} & 0.5 & 6.7292 & 6.7170 \\
\hline \multirow{3}{*}{0.75} & 1 & 5.6213 & 5.6198 \\
\hline & 2 & 4.4498 & 4.4429 \\
\hline & 0.5 & 5.9178 & 5.9238 \\
\hline \multirow{2}{*}{1} & 1 & 5.1256 & 5.1301 \\
\hline & 2 & 4.1903 & 4.1887 \\
\hline & 0.5 & 5.3505 & 5.3583 \\
\hline & 1 & 4.7612 & 4.7496 \\
\hline
\end{tabular}

Table 3: Comparison between $\mathrm{HA}$ results and exact solution for various $\alpha, \omega_{0}^{2}$.

To illustrate and verify the accuracy of Hamiltonian Approach (HA), the comparison between the approximate period $\left(T_{H A}\right)$ with the exact one [13] $\left(T_{\text {for }}\right)$ are tabulated in Table 3 for various values of $\alpha$ and $\omega_{0}^{2}$ when $A=1$

In Figure 1 the approximate dimensionless deflection of Euler Bernoulli beam for each dimensionless time have been compared to exact solution for case of $\alpha=0.15, \omega_{0}^{2}=0.5$ and $A=3$ as it can be illustrated in Figure 1 the exact solutions have a good agreement with approximate analytical results.

\section{Conclusion}

In this paper, we have used analytical methods called Differential Transformation Method (DTM) and Hamiltonian Approach (HA) to determine the approximate solution of Euler Bernoulli beam nonlinear vibration which is subjected to the axial force.

The comparison between analytical approximate results and exact integral solutions assures us about the convenience and accuracy of the solution procedure. Some patterns are also given to illustrate the effectiveness and convenience of the methodologies. 
Citation: Azimi M, Kariman S (2013) Periodic Solution for Vibration of Euler-Bernoulli Beams Subjected to Axial Load Using DTM and HA. J Appl Mech Eng 2: 125. doi:10.4172/2168-9873.1000125

\section{References}

1. Timoshenko SP, Young DH, Weaver W (1990) Vibration Problems in Engineering. John Willey \& Sons, New York, USA.

2. Pirbodaghi T, Ahmadian MT, Fesanghary M (2009) On the Homotopy Analysis Method for Nonlinear Vibration of Beams. Mechanics Research Communications 36: 143-148.

3. Ozturk B (2009) Free Vibration Analysis of Beam on Elastic Foundation by Variational Iteration Method. Int J Nonlinear Sci Numer Simulation 10: 12551262.

4. Adomian G (1990) A Review of the Decomposition Method and Some Recent Results for Nonlinear Equation. Mathematical Computation and Modelling 13: $17-43$

5. Ganji DD, Azimi M, Mostofi M (2012) Energy Balance Method and Amplitude Frequency Formulation Based of Strongly Nonlinear Oscillators. Ind J Pure Ap Phy 50: 670-675

6. Hsu JC, Lai HY, Chen CK (2009) An Innovative Eigenvalue Problem Solve For Free Vibration of Timoshenko Beams By Using the Adomian modified Decomposition Method. J Sound Vib 325:451-470.
7. Xu L (2011) A Hamiltonian Approach for a Plasma Physics Problem. Computers \& Mathematics with Applications 61:1909-1911

8. Bervillier C (2012) Status of Differential Transformation Method. Applied Mathematics and Computation 218: 10158-10170.

9. Chen SS, Chen CK (2009) Application of Differential Transformation Method to the Free Vibrations of Strongly Nonlinear Oscillators. Nonlinear Analysis: Real World Applications 10: 881-888.

10. Barari A, Khaliji HD, Ghadimi M, Domairry G (2011) Nonlinear Vibration of Euler Bernoulli Beams. Latin American Journal of Solids and Structures 8: 139-148.

11. Ganji DD, Azimi M (2012) Application of Max Min Approach and Amplitude Frequency Formulation to Nonlinear Oscillation method for some nonlinear oscillation problems. U.P.B. Sci Bull 74: 131-140.

12. Liu Y, Gurram CS (2009) The Use of He's Variational Iteration Method for Obtaining the Free Vibration of an Euler Bernoulli Beam. Mathematical and Computer Modelling 50: 1545-1552.

13. Marinca V, Herisanu N (2006) A modified iteration perturbation method for some nonlinear oscillation problems. Acta Mechanica 184: 231-242. 